\title{
The molecular evolution of PL10 homologs
}

\author{
Ti-Cheng Chang 1,2 and Wan-Sheng Liü1,2
}

\begin{abstract}
Background: $P L 10$ homologs exist in a wide range of eukaryotes from yeast, plants to animals. They share a DEAD motif and belong to the DEAD-box polypeptide 3 (DDX3) subfamily with a major role in RNA metabolism. The lineagespecific expression patterns and various genomic structures and locations of PL10 homologs indicate these homologs have an interesting evolutionary history.

Results: Phylogenetic analyses revealed that, in addition to the sex chromosome-linked PL10 homologs, DDX3X and DDX3Y, a single autosomal PL10 putative homologous sequence is present in each genome of the studied non-rodent eutheria. These autosomal homologous sequences originated from the retroposition of DDX3X but were pseudogenized during the evolution. In rodents, besides $D d x 3 x$ and $D d x 3 y$, we found not only P/10 but another autosomal homologous region, both of which also originated from the $D d x 3 \times$ retroposition. These retropositions occurred after the divergence of eutheria and opossum. In contrast, an additional X putative homologous sequence was detected in primates and originated from the transposition of DDX3Y. The evolution of PL10 homologs was under positive selection and the elevated $\mathrm{Ka} / \mathrm{Ks}$ ratios were observed in the eutherian lineages for DDX3Y but not $P L 10$ and DDX3X, suggesting relaxed selective constraints on DDX3Y. Contrary to the highly conserved domains, several sites with relaxed selective constraints flanking the domains in the mammalian PL10 homologs may play roles in enhancing the gene function in a lineage-specific manner.

Conclusion: The eutherian DDX3X/DDX3Y in the X/Y-added region originated from the translocation of the ancient $P L 10$ ortholog on the ancestral autosome, whereas the eutherian PL10 was retroposed from DDX $3 X$. In addition to the functional PL10/DDX3X/DDX3Y, conserved homologous regions on the autosomes and X chromosome are present. The autosomal homologs were also derived from DDX3X, whereas the additional X-homologs were derived from DDX3Y. These homologs were apparently pseudogenized but may still be active transcriptionally. The evolution of PL10 homologs was positively selected.
\end{abstract}

\section{Background}

PL10 was first identified in mouse by using a human Y chromosome (Chr) derived probe [1] and is present in a wide range of eukaryotes from yeast, plants, and animals, including humans [2]. In the mouse, $P l 10$ has been shown to encode a functional protein with an important DEAD motif (Asp-Glu-Ala-Asp), which plays essential roles in spermatogenesis [3]. In eutherian mammals, PL10 has two closely-related paralogs, $D D X 3 X$ (DEAD box polypeptide 3, X-linked) and DDX3Y (DEAD box polypeptide 3, Y-linked), located on the sex Chrs. PL10, DDX3X and $D D X 3 Y$ share the DEAD motif and constitute the DDX3/ DED1 (ATP-dependent DEAD-box RNA helicase) sub-

* Correspondence: wul12@psu.edu

1 Department of Dairy and Animal Science, The Center for Reproductive Biology and Health (CRBH), College of Agricultural Sciences, The Pennsylvania State University, University Park, PA, 16802, USA

Full list of author information is available at the end of the article family under the DEAD-box helicase family [4] with a major function related to RNA metabolism [5]. The $D D X 3 / D E D 1$ subfamily is involved in diverse cellular process including tissue differentiation at distinct developmental stages, embryogenesis, asexual reproduction, cell regeneration, tumorigenesis and immune response $[2,6-8]$, which have been reviewed comprehensively by Rosner et al. [2].

Interestingly, the biological roles of the eutherian members in $D D X 3$ subfamily appear to be varied and lineagedependent although they share domain structures and highly similar sequences. In eutheria, the $D D X 3 X$ has been shown to elicit immunoresponse because the $D D X 3 X$ can interact with TANK-binding kinase 1 (TBK1) to induce the type I interferon (IFN) promoter and the downstream immune pathway [6]. In addition, $D D X 3 X$ also plays a role in HIV infection and becomes an impor- 
tant target in antiviral therapy [9]. On the other hand, the human DDX3Y lies within the azoospermia factor a $(A Z F a)$ region on the proximal Yq11.21 and the deletion of human $D D X 3 Y$ resulted in the oligozoospermia, azoospermia and the male sertoli-cell only syndrome $[10,11]$. In spite of their high amino acid (aa) similarity (91\%), $D D X 3 X$ cannot rescue the loss-of-function of $D D X 3 Y$ in human [2], signifying the functional diversification between $D D X 3 X$ and $D D X 3 Y$. The human $D D X 3 Y$ is believed to be one of the essential genes involved in human spermatogenesis and male fertility [12]. In contrast to the human ortholog, the pivotal role of $D d x 3 y$ in spermatogenesis has been replaced by the autosomal Pl10 in mice [3]. The mouse Pl10 is believed to evolve from $D d x 3 x$ through the retroposition mechanism [13]. More interestingly, the bovine PL1O has also been proved to be active at the transcription level even though it may lose protein-coding potential [14]. In addition to the lineagedependent functionality of $P L 10$, the tissue specificity of $D D X 3 X / D D X 3 Y$ homologs has also been shown to vary in mouse and human [15,3]. The lineage-specific expression patterns and the diverse genomic structures and locations of PL10 homologs suggest that the PL1O homologs regulate biological process via divergent mechanisms and evolved differently. However, previous studies focused mainly on elucidating the function rather than the evolution of PL10, which elicited our interest to investigate the evolutionary history behind $P L 10, D D X 3 X$, and $D D X 3 Y$. Here, we report the results from a phylogenetic analysis of the PL10 homologs in 19 different species.

\section{Results}

\section{The identification of PL10 homologous sequences}

To obtain deep insight into PL1O evolution, we collected the PL10 related genes deposited in NCBI [16] and detected its potential homologs by comparing the mouse Pl10 mRNA sequence against the UCSC genome database [17]. In addition to the 22 annotated sequences for PL10, DDX $3 X$ and DDX3Y, we identified 15 PL10 putative homologous regions (coverage $>50 \%$ ) in the genomes of mammals (Table 1). These putative homologs occupied the genomes with two major patterns in terms of their spanning size $(2 \sim 4$ and 10 14 Kb). The large-size homologs are located in the sex Chrs containing intronexon structures, while the small-size ones are mostly autosomal and intronless (Table 1).

We extracted the sequences from these putative homologous regions and conducted a gene prediction using GENSCAN [18] to identify whether these homologous sequences maintain protein-coding potential. Based on gene similarity, structures and chromosomal locations, we obtained the predicted DDX3X in chimp and orangutan, and Pl10 on Chr 13 in rat (RNO13). The predicted chimp and orangutan DDX3X matched the predicted coding proteins in ENSEMBL [19] (Table 1). Compared to the human DDX3X protein of 662 aa, the predicted peptide is much shorter in the chimp with only 438 aa because of the incomplete sequence. The predicted rat Pl10 matched to the entry, NP_001102328.1, in the NCBI database, and we concluded that it is the rat Pl10 based on its intronless structure and high sequence similarity (96\%) with the mouse Pl10. In opossum, the predicted DDX3 peptide matched to ENSMODT00000026845 in ENSEMBL [19] (Table 1). The remaining 11 homologs either do not have an open reading frame (ORF) or have a premature stop codon (Table 2). Thus, they are pseudogenes.

\section{The analyses of PL10 phylogeny}

Using the 22 PL10 related entries from NCBI together with 15 previously-described putatively homologous sequences, we constructed a phylogenetic tree to investigate the evolutionary relationship among these homologs. The tree clearly indicated several evolutionary clusters (Fig. 1). The first cluster is the PL10/DDX3X cluster, within which the putative homologous sequences on primate Chr4 were in the same clade and clustered with the primate $D D X 3 X$. The autosomal homologous regions in ruminants and carnivores, including the bovine $P L 10$ pseudogene [14], were also in the same cluster and grouped with the $D D X 3 X$ counterparts as in primates (Fig. 1). No apparent insertions were detected in these homologous regions. The mouse and rat $P l 10$ were in the same branch. However, an additional putative homolog of $D D X 3 X$ was detected in mouse (MMU1) and rat (RNO19), which was grouped with its corresponding $D D X 3 X$ gene, respectively, before clustering them together into a single group. It is noteworthy that all homologs of $D D X 3 X$ identified in mammals are intronless (Table 1). Since the mammalian $D D X 3 X$ contains an intron-exon structure, we reasoned that these intronless homologs are most likely the evolutionary trace after the $D D X 3 X$ retroposition.

In addition to the functional eutherian $D D X 3 X$, we detected another putative homologous region on primate ChrX which was present on the same branch with the $D D X 3 Y$ instead of the $D D X 3 X$ (Fig. 1). In contrast to the autosomal homologs, these $\mathrm{ChrX}$ putative homologs contain one or more insertions that appear to fit with the typical GT/AG splicing rule (Table 2), raising the possibility that these additional $\mathrm{X}$ homologs may have derived from a transposition event before the primate divergence (Fig. 1). Furthermore, in opossum and chicken, only a single homologous region with the intron-exon structure was detected on opossum Chr4 (MDO4) and chicken Chr1 (GGA1), respectively (Table 1). 
Table 1: PL10 homologs in 19 species.

\begin{tabular}{|c|c|c|c|c|c|c|}
\hline Species (build version) & Chr & Gene & Homologous region & & Span & Accession Number \\
\hline \multirow[t]{4}{*}{ Human (37.1) } & Y & $D D \times 3 Y$ & 15016838 & 15030444 & 14229 & NM_004660.3 \\
\hline & $x$ & $D D \times 3 X$ & 41193484 & 41207386 & 14657 & NM_001356.3 \\
\hline & $x$ & & 73340837 & 73351755 & 18744 & \\
\hline & 4 & & 104493233 & 104495627 & 3122 & \\
\hline \multirow[t]{4}{*}{ Chimp (2.1) } & Y & $D D X 3 Y$ & 18024925 & 18030276 & 5352 & NM_001008986.1 \\
\hline & $x$ & $D D X 3 X$ & 41567709 & 41578379 & 10637 & ENSPTRT00000048707 \\
\hline & $x$ & & 73472677 & 73479677 & 7000 & \\
\hline & 4 & & 106890486 & 106891981 & 1496 & \\
\hline \multirow[t]{3}{*}{ Orangutan (2.0.2) } & $x$ & $D D X 3 X$ & 41920594 & 41934087 & 13494 & ENSPPYT00000023631 \\
\hline & $x$ & & 71584239 & 71585574 & 1336 & \\
\hline & 4 & & 108027398 & 108029797 & 2400 & \\
\hline \multirow[t]{4}{*}{ Mouse (37) } & Y & $D d x 3 y$ & 599654 & 615438 & 15785 & NM_012008.1 \\
\hline & $x$ & $D d x 3 x$ & 12858220 & 12869030 & 11577 & NM_010028.3 \\
\hline & 1 & Pl10 & 188791295 & 188794506 & 3212 & NM_033077.2 \\
\hline & 1 & & 28046742 & 28049045 & 2304 & \\
\hline \multirow[t]{3}{*}{ Rat (RGSC 3.4) } & $x$ & $D d x 3 x$ & 21497214 & 21508627 & 11414 & XM_228701.4 \\
\hline & 13 & Pl10 & 103083154 & 103086327 & 3174 & NM_001108858.1* \\
\hline & 19 & & 5498280 & 5501578 & 3299 & \\
\hline \multirow[t]{2}{*}{$\operatorname{Dog}(2.0)$} & $x$ & $D D X 3 X$ & 35708607 & 35722852 & 14282 & XM_856175.1 \\
\hline & 22 & & 15373292 & 15375479 & 2188 & \\
\hline \multirow[t]{2}{*}{ Horse (EquCab2.0) } & $x$ & $D D X 3 X$ & 33503944 & 33514664 & 10721 & XM_001491432.2 \\
\hline & 17 & & 31837579 & 31839780 & 2202 & \\
\hline \multirow[t]{3}{*}{ Cow (Btau_4.0) } & $\mathrm{Y}$ & $D D \times 3 Y$ & $86^{\Delta}$ & $5279 \Delta$ & 5194 & {$[14]$} \\
\hline & $x$ & $D D X 3 X$ & 68833 & 82540 & 13708 & {$[14]$} \\
\hline & 15 & PL10 & 186070 & 189757 & 3688 & {$[14]$} \\
\hline Opossum (MonDom5) & 4 & $D D \times 3$ & 22331869 & 22343917 & 12049 & $\begin{array}{l}\text { ENSMODT0000002684 } \\
5\end{array}$ \\
\hline Chicken (2.1) & 1 & $D D \times 3$ & 115610539 & 115617788 & 7250 & NM_001030800.1 \\
\hline X. tropicalis (4.1) & & $D D \times 3$ & 940555 & 947979 & 7425 & BC063374 \\
\hline Zebrafish (Zv7) & 6 & PL10 & 25945 & 42249 & 16304 & NM_130941 \\
\hline Clamworm & & PL10a & & & & AM048813.1 \\
\hline Flatworm & & $D j V L G A$ & & & & АВ017002.1 \\
\hline Hydra & & CnPL10 & & & & AB047381.1 \\
\hline Rice & & $\begin{array}{l}\text { DEAD-box RNA } \\
\text { Helicase }\end{array}$ & & & & NM_001074753.1 \\
\hline Arabidopsis & & $\begin{array}{l}\text { DEAD-box RNA } \\
\text { Helicase }\end{array}$ & & & & NM_129813.4 \\
\hline Fission Yeast & & $D E D 1$ & & & & AJ237697.1 \\
\hline \multirow[t]{2}{*}{ Yeast } & & $D B P 1$ & & & & X55993.1 \\
\hline & & DED1 & & & & X57278.1 \\
\hline
\end{tabular}

* The corresponding protein entry is NP_001102328.1.

$\Delta$ The position was annotated based on NW_001496707.1. 
Table 2: Pairwise comparison between mouse PI10 (mPI10) and the non-annotated homologous regions in eutheria.

\begin{tabular}{|c|c|c|c|c|c|}
\hline \multicolumn{2}{|c|}{$\begin{array}{l}\text { Genomic Position of Non-annotated } \\
\text { PL10 Homologs }\end{array}$} & \multirow{2}{*}{$\begin{array}{l}\begin{array}{l}\text { Identity with } \mathbf{m} \\
\text { PI10 (\%) }\end{array} \\
80.08 \\
\end{array}$} & \multirow{2}{*}{$\begin{array}{l}\begin{array}{l}\text { Alignment Cover- } \\
\text { age with } \mathbf{m} \text { PI10 (\%)* }\end{array} \\
100.00\end{array}$} & \multirow{2}{*}{$\begin{array}{c}\text { Aligned Segment Number } \Delta \\
1\end{array}$} & \multirow{2}{*}{$\begin{array}{l}\text { Putative Peptide } \\
\text { Length (aa)\# } \\
84\end{array}$} \\
\hline Human Chr4 & (HSA4) & & & & \\
\hline Human ChrX & (HSAX) & 78.52 & 98.38 & 2 (Ins: 8958 bp) & $241(\mathrm{DDX} 3 \mathrm{Y})$ \\
\hline Chimp Chr4 & (PTR4) & 74.83 & 81.93 & 1 & 84 \\
\hline Chimp ChrX & (PTRX) & 78.37 & 98.34 & 2 (Ins: 1134 bp; Ns: 3010 bp) & 472(DDX3X) \\
\hline Orangutan $\mathrm{Chr} 4$ & (PPY4) & 79.80 & 74.18 & 2 (Ns: 463 bp) & 132 \\
\hline Orangutan $\mathrm{ChrX}$ & (PPYX) & 80.94 & 67.42 & 1 & 124(DDX3Y) \\
\hline Mouse Chr1 & (MMU1) & 79.45 & 95.41 & 1 & 349(DDX3X) \\
\hline Rat Chr19 & (RNO19) & 80.27 & 51.08 & $\begin{array}{l}3 \text { (Ns: } 1509 \text { bp; Ins: } 316 \text { bp; } \\
\text { Gap: } 962 \text { bp) }\end{array}$ & 192(DDX3X) \\
\hline Dog Chr22 & (CFA22) & 73.00 & 58.00 & 1 & N/A \\
\hline Horse Chr17 & (ECA17) & 75.98 & 96.82 & 1 & $\mathrm{~N} / \mathrm{A}$ \\
\hline Cow Chr15 & (BTA15) & 81.00 & 72.52 & 1 & $\mathrm{~N} / \mathrm{A}$ \\
\hline
\end{tabular}

* The alignment coverage was calculated based on the pairwise alignment between the mouse P/10 and identified homologous regions.

$\triangle$ Ns: the homologous region contains incomplete sequences. Ins: the homologous region is interrupted by non-homologous sequences. Gap: part of $\mathrm{mPl} 10$ was not alignable with the detected homologous sequences.

\#The peptides were predicted via GENSCAN [18]. The protein name in parenthesis indicated the matched entries with lowest e-value in blastp analysis. N/A: not applied.

\section{Positive selection test for the PL10 related genes}

We compared the one-ratio model with the free-ratio model to test the lineage-specific positive selection for the PL1O related functional homologs in our dataset using PAML4 package [20]. The one-ratio model assumes the same $\mathrm{K}_{\mathrm{a}} / \mathrm{K}_{\mathrm{S}}(w)$ ratio for all the lineages. The log-likelihood value under this model was $l_{0}=-22217.097$ with 58 parameters where the transition/transversion rate ratio was $k=1.685$ and $w=0.041$. The $w$ was computed as the average of all codon sites and lineages. The free-ratio model assumes an independent $w$ ratio for each branch and the number of parameters was increased to 104 for our dataset in this model. The likelihood value under this model was $l_{1}=-22105.980$. The comparison of the likelihood value, $2 \Delta l=2\left(l_{1}-l_{0}\right)$, was 222.234 as determined by the $X^{2}$ distribution with degree of freedom (df) of 46 (p < 0.001 ), allowing us to reject the one-ratio model and conclude that the $w$ ratios are varied among lineages (Fig. 2). In mammals, the estimates of $w$ ratios were all lower than 0.1 on the branches leading to the lineages for PL10 and $D D X 3 X$, whereas the $w$ ratios were higher on average (0.5) among the lineages for $D D X 3 Y$ (Fig. 2). Furthermore, the primate lineages for $D D X 3 Y$ in human and chimp were detected to be subject to positive selection (Fig. 2). The branches leading to the mammalian PL1O homologs clade (Fig. 2) also showed $w$ ratios larger than 1 , suggesting that the evolution of PL10/DDX3X/DDX3Y was under positive selection.
Since some lineages were positively selected, especially in the case of $D D X 3 Y$, we further used a small dataset containing only the mammalian homologous coding sequences to examine the positively selected sites. The test statistic of likelihood ratio test (LRT) between the one-ratio model (M0) and the discrete model (M3) was 128.182 that is greater than the critical value $\chi_{1 \%}^{2}=13.28$ when $\mathrm{df}=4$ [Additional File 1 and File 2]. This suggested that the selective pressure is diverse among the codons. Three site classes calculated under model M3 have prior probability of $\mathrm{p}_{0}=0.887, \mathrm{p}_{1}=0.108$, and $\mathrm{p}_{3}=0.005$ with the $\mathrm{Ka} / \mathrm{Ks}$ ratios of $w_{0}=0.025, w_{1}=0.316$ and $w_{2}=2.549$ [Additional File 1]. The posterior probabilities of site classes calculated in model M3 are shown in Fig. 3. However, the LRT of the other two pairs of models, M1a (Nearly Neutral)/M2a (Selection) and M7 (beta)/M8 (beta \& w), generated an incongruent result. The test statistic of the M1a/M2a is insignificant ( $>1$ ), whereas the M7/M8 generated a significant result with a LRT value of 16.97 greater than the critical value at $\mathrm{df}=2, \chi_{1 \%}^{2}=9.1$ $(\mathrm{p}<0.01)$ [Additional File 1 and File 2], which together gave rise to the marginal prediction of the codon sites with relaxed selective constraints. Four (9A, 10L, 24S, 425S) and six (9A, 10L, 24S, 425S, 608A, 609S) sites were inferred to contain increased $w$ ratios under models M2a and M8, respectively. Four of the six inferred sites in model M8 coincided with the result of model M2a, 


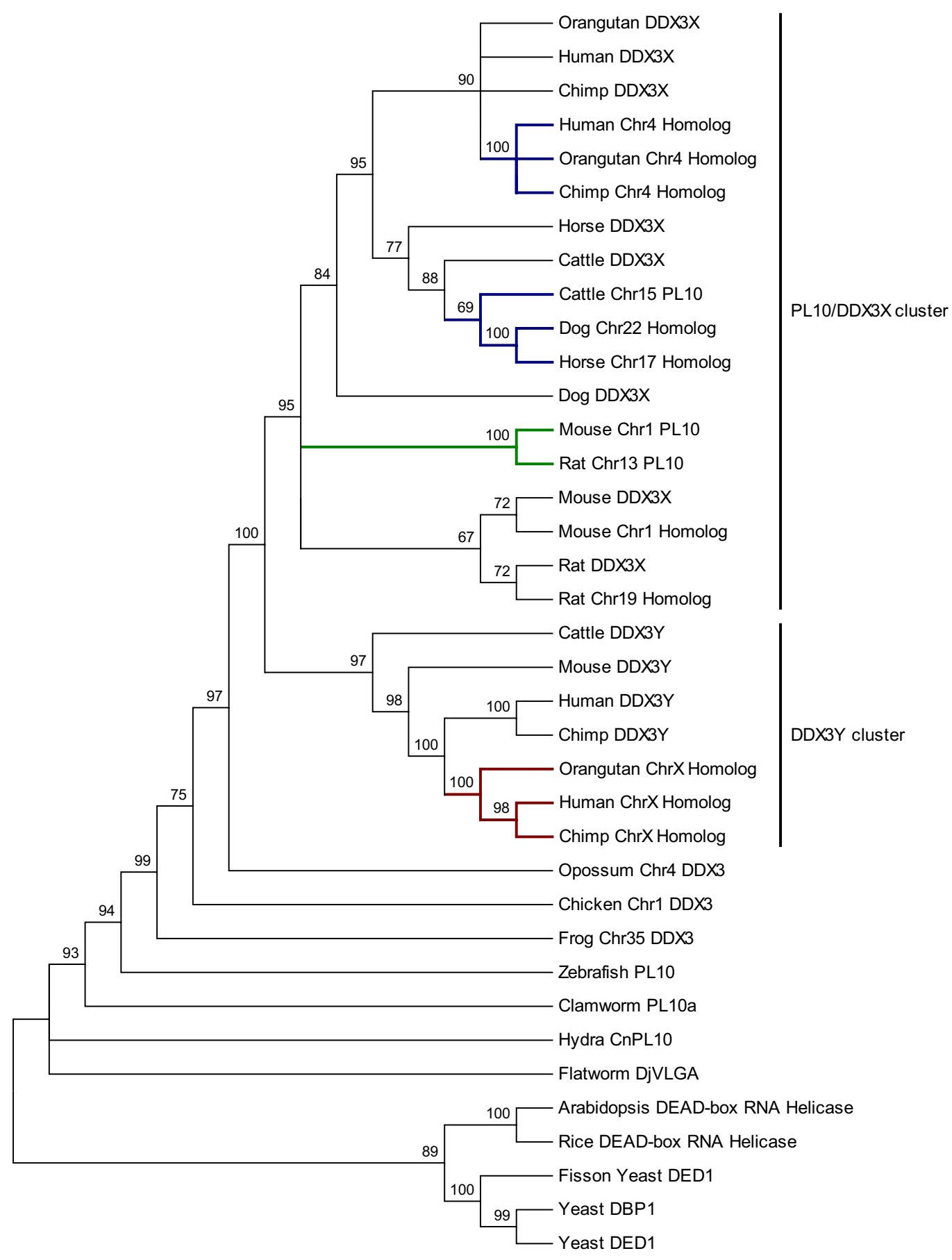

Figure 1 The bootstrap consensus tree of PL10 homologous sequences. The evolutionary tree was built based on the Neighbor-Joining method implemented in MEGA4 $[55,62]$. The bootstrap consensus tree is inferred from 1000 replicates and the branches corresponding to partitions reproduced in less than $65 \%$ bootstrap replicates are collapsed. The bootstrap values are shown as percentages next to the branches. The evolutionary distances were computed using the Maximum Composite Likelihood method [63] and in the units of the number of base substitutions per site. The rate variation among sites was modeled with a gamma distribution (shape parameter $=0.91$ ). All positions containing alignment gaps and missing data were eliminated by pairwise deletion. A total of 3944 positions were in the final dataset [Additional File 6]. The branches leading to the non-annotated autosomal homologous clusters of PL10 are highlighted in blue; the branches leading to the rodent P/10 are highlighted in green; the branches leading to the non-annotated X-homologs are highlighted in red. The PL10/DDX3X cluster and the DDX3Y cluster are marked by vertical lines on the right. 


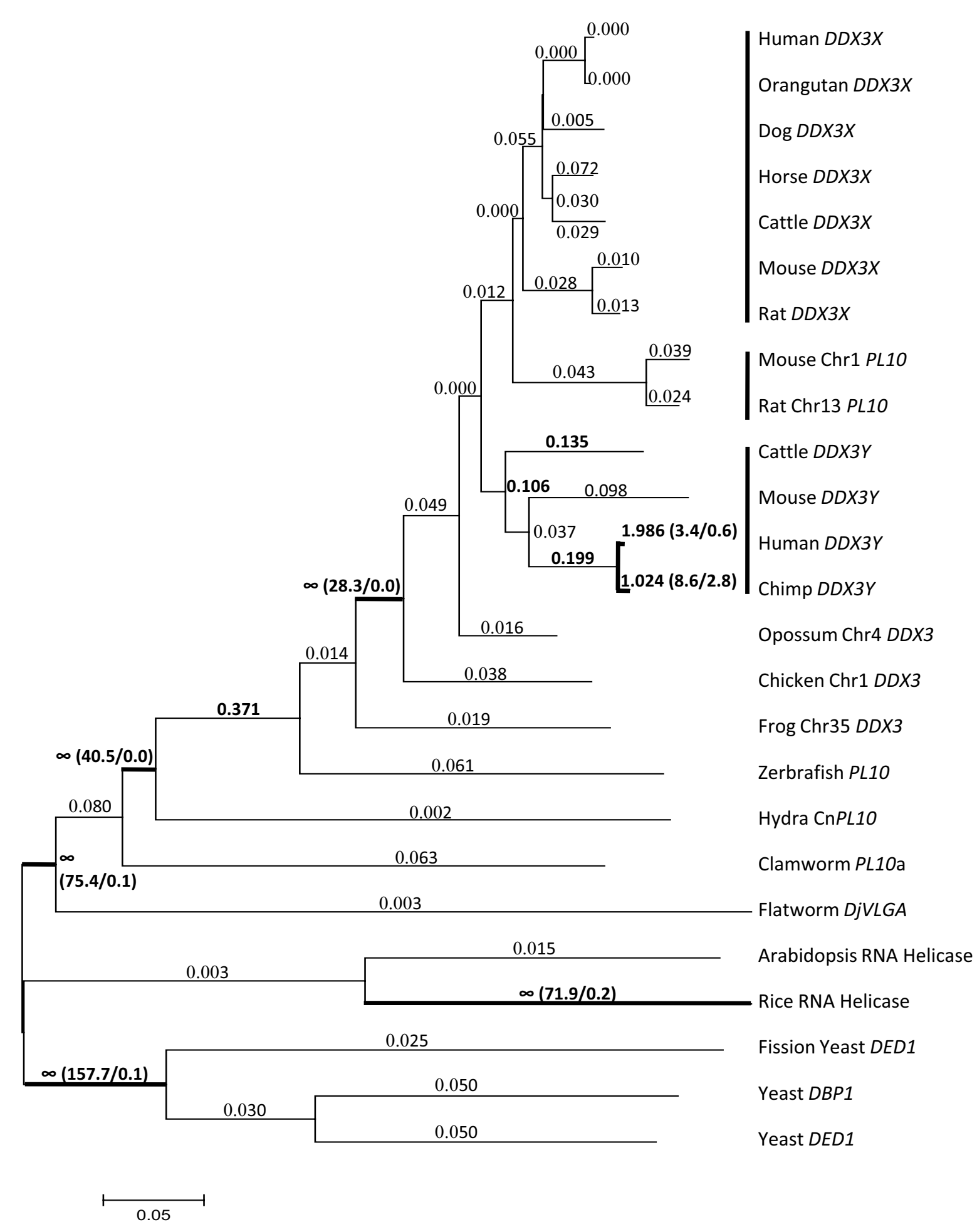

Figure 2 The tree of the DDX3 family established for the positive selection test based on Maximum Likelihood approach. The branch length was estimated in the unit of the number of nucleotide substitutions per nucleotide. Values larger than 0.1 are denoted in bold. The numbers in the parenthesis represent the estimated numbers of nonsynonymous substitutions against synonymous substitutions of the specific branch. Scale bar = 0.05 unit. 


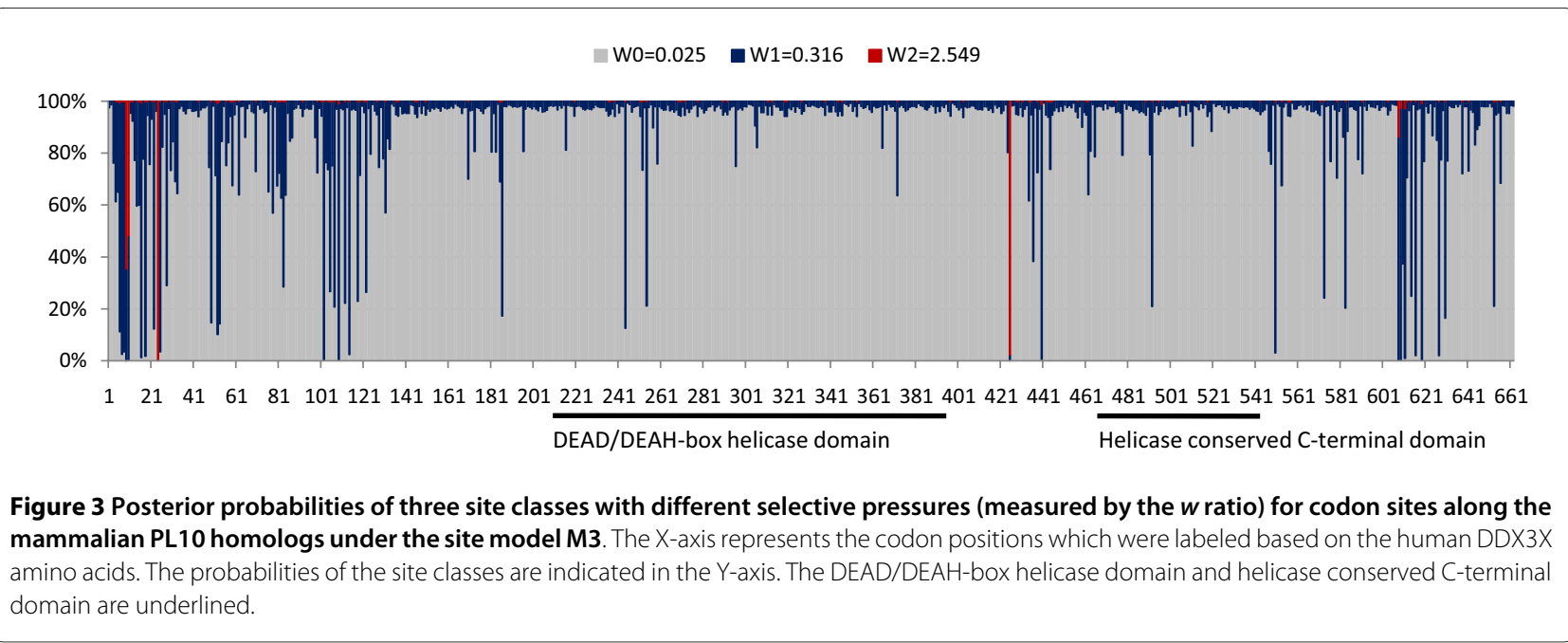

including Ala9, Leu10, Ser24, Ser425, in which the Ser24 and Ser425 have posterior probability higher than 0.9 under model M8 [Additional File 1]. All of the inferred sites are located in the non-domain regions.

\section{Conservation of PL10 homologs}

We conducted a multiple alignment for all the analyzed sequences to investigate the domain conservation in PL10, DDX3X and DDX3Y, and found that the DEAD/ DEAH box helicase domain (Pfam: PF00270) and helicase conserved C-terminal domain (Pfam: PF00271) of the $D D X 3$ genes are highly conserved [Additional File 3]. We evaluated the degree of conservation by ConSurf (Fig. 4A) [21], which assigned the conservation score to each site of the provided DDX3X structures (PDB: 2I4I) [22] based on the empirical Bayesian method [21]. After mapping the conservation score to the structure, we found that the highly conserved codons concentrated in the cleft where the adenosine monophosphate (AMP) and RNA substrates interact with the DDX3 proteins (Fig. 4).

\section{Discussion}

In the non-eutherian lineages, $P L 10$ is the sole member of the DDX3 subfamily, whereas in eutheria, the ancient PL10 gene is located on the ancestral sex Chrs, resulting in the sex Chr-linked orthologs, $D D X 3 X$ and $D D X 3 Y$ (Fig. 1). Molecular evolutionary studies in recent years have established that the mammalian sex Chrs originated from a pair of ordinary autosomes, and most ancestral genes on that pair were still maintained on the $\mathrm{X}$ Chr but degenerated on the Y Chr due to the lack of recombination [23-25]. However, the Y Chr intends to maintain the functional genes that are beneficial to the male, such as those genes involved in spermatogenesis including $D D X 3 Y$ [26]. Like the non-eutherian PL10, DDX3X and $D D X 3 Y$ comprise the intron-exon structures, supporting the concept that $D D X 3 Y$ and $D D X 3 X$ are the evolutionary relics of the ancestral autosomal PL10. In opossum, the PL10 homologous sequence was detected only on MDO4 but not on the sex Chrs. The opossum Chr4 homolog also contains the intron-exon structure with predicted peptide close to $D D X 3 X$. Similarly, the single homologous sequence detected in chicken was located on the autosomes and it contains introns. A recent study for the gene cluster in the $\mathrm{X} / \mathrm{Y}$-added region of mammalian sex Chrs, $\mathrm{XAR}$ and YAR, revealed that the gene cluster and the gene order of this region are the same on chicken GGA1 but separated on opossum MDO4 and MDO7 [15], suggesting a single translocation event gave rise to the different chromosomal locations of the gene cluster among chicken and opossum. The $D D X 3 X / D D X 3 Y$ also reside

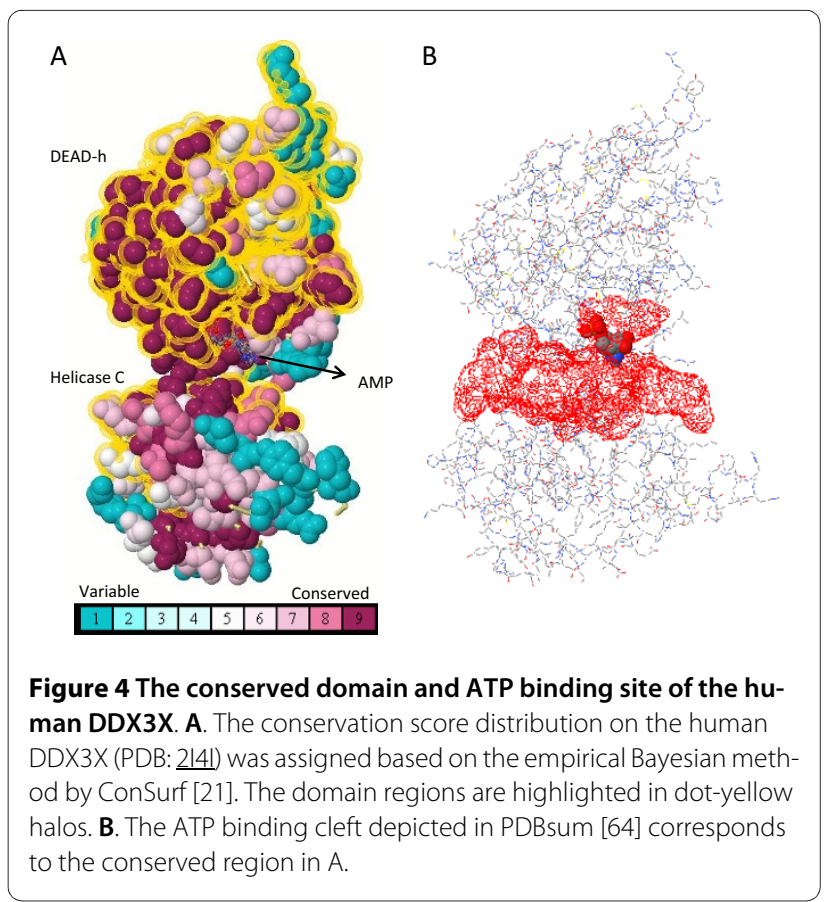


within the XAR/YAR, which allowed us to reach the parallel conclusion that the translocation generated the PL10 homologs on the chicken Chr1 and opossum Chr4, and $D D X 3 X / D D X 3 Y$ on the eutherian sex Chrs (Table 1, Fig. $1)$.

Of particular interest is the occurrence of the mouse $P l 10$, an intronless gene and the only demonstrated functional autosomal ortholog in mammals to date. Consistent with a previous deduction [13], our result supported that the rodent $P l 10$ was derived from the retroposition of the $D D X 3 X$ genes [27]. Retroposition is a crucial mechanism of gene duplication [28] and generates many new genes in new genomic positions through the reverse transcription of a parental gene $[27,29,30]$. The parental gene usually contains introns, whereas the processed retrocopy is intronless [27]. Thus, the other detected putative autosomal PL1O homologous sequences without apparent intron-exon structure in cattle, horse, dog and primates may have also evolved through the retroposition mechanism. These intronless homologous sequences on autosomes were consistently detected in eutherians but not in opossum, suggesting that the retroposition occurred after the divergence of eutherian and other mammals around 150 to 170 million years ago [31]. This raised an interesting question, why does the autosomal retroposition of the PL10 occur specifically in eutheria? It may be partially explained by the important functional role of mouse Pl1O and the recently discovered bovine PL10. The mouse Pl10 has been evidenced to be a central gene regulating the spermatogenesis and replace the role of DDX3Y [3]. The bovine PL10, albeit pseudogenized during the evolution, has also been proven to be active transcriptionally and may be involved in the regulatory coordination of bovine spermatogenesis [14]. Although the coding potential of the autosomal PL1O homologous sequences in eutheria, except in mouse, is diminished, we cannot exclude the possibility that these homologous sequences may be involved in regulating some biological process at the transcriptional level. Indeed, previous studies suggested that the pseudogenes may regulate the expression of the functional paralogous genes by producing antisense RNA [32,33]. Therefore, it is valuable to investigate whether these homologous sequences are transcriptable and their potential function in the future.

The maximum likelihood ratio test (LRT) for different lineages indicated that the $\mathrm{Ka} / \mathrm{Ks}$ ratios in the PL1O homologs are varied among the evolutionary lineages. The $\mathrm{Ka} / \mathrm{Ks}$ ratio along the branches among the mammalian lineages showed that the evolution of the mammalian PL10 homologs were not subject to positive selection, except for the human and chimp DDX3Y that are positively selected $(w>1)$. In addition to the chimp and human $D D X 3 Y$, we found that the ratios for the other eutherian lineages for $D D X 3 Y$ appear to be higher when compared to those for mammalian PL1O and DDX3X, which is in line with a finding by Wilson and Makova [15]. These elevated $w$ ratios can be explained by either the effect of relaxed selective constraints for the lineages containing $D D X 3 Y$ due to the absent recombination of the ChrY or a weak positive selection operating on the Yhomologs [20]. The latter may still continue to refine the male-specific function for the Y-homologs [34]. In contrast, the mammalian lineages for DDX $3 X$ and PL1O with extremely low $\mathrm{w}$ ratios suggested that purifying selection may act strongly on the mammalian PL1O and Xhomologs. Furthermore, the $w$ ratios of the branches leading to the avian and mammalian lineages were larger than 1, indicating that the emergence of eutherian PL10 homologs was selected positively to acquire species-specific gene function and purifying selection acted on the $D D X 3 X$ and PL10 homologs to preserve their crucial biological function and avoid their divergence.

A $D D X 3 X / D D X 3 Y$-specific multi-residue insertion (EALRAMKENG) has been observed to form an important positively charged cavity with the neighboring positive residues to increase the RNA binding surface in humans [35]. After incorporating PL10 and the homologous regions, we found that the insertion was highly conserved in the PL10 homologs of fish, frog, chicken, and other mammals, suggesting that the functional constraints occurred along the cavity region in the related homologs. Conversely, this insertion was not well-conserved in plants and invertebrates. Furthermore, the $D D X 3 X$ displayed no activity with the RNA substrate when different flanking regions surrounding the domains were removed [35]. A similar effect was proven in other DEAD-box helicase related genes, such as the UAP56 [36] and DP103 [37], where the deletion of either the Nterminal or C-terminal flanking sequences outside the domain core regions impacts their helicase and ATPase activity, signifying the regulatory roles of the flanking regions. As shown from the analysis of positively selected amino acids, all the marginally inferred selected sites were located in the non-domain regions (Fig. 3) and may have served as the targets for improving the gene function during the evolution. One of the inferred sites, Ser425, occurred in the hinge region between two domains, suggesting its potential role in the adaption of the PL10 protein conformations and ligand-binding specificity. The slightly elevated $w$ ratios were observed mostly in the non-domain regions as depicted in Fig. 3, indicating the purifying selection may attenuate in these regions to allow the functional accommodations of the PL10 homologs. Moreover, several sites in the flanking regions of the human DDX3X have been shown to undergo epigenetic modifications, including Ser2[38], Tyr69[39,40], Ser74[41], Ser76[41], Ser78[41], Tyr104[39], Ser125[41], Ser590[42], Ser594[43,42] and 
Ser612[43]. Meanwhile, two sites in the flanking sequences of the human DDX3Y, Tyr69 [39,44] and Ser592 [45], have been shown to be phosphorylated, and they are conserved with the modified sites at Tyr69 and Ser594 in the human DDX3X. The mouse Pl10 also has two phosphorylated sites at Tyr282 and Tyr465, but both of them were located in the domains [46,47]. The sequence comparison showed that the selection force has limited the divergence in the regions flanking the domains of PL10 related genes in fish, frog, bird and mammals, and most of the epigenetically modified sites were highly conserved among these species. Interestingly, despite the high degree of conservation, Ser76 exists specifically in the PL10 and DDX3X homologs but not in the DDX3Y orthologs. This distinction and different epigenetic modification pattern may partly contribute to the functional divergence between the eutherian $D D X 3 Y$, $D D X 3 X$ and PL10. The functional specificity of PL10 homologs appear to be determined multifactorially, including the sequence elements located in the non-conserved regions, the factors controlling the diverse temporal and spatial expression patterns [14], and the distinct epigenetic modification patterns [35].

This study was limited to the availability of complete genomes and the accuracy of the genome assembly. Even though the number of finished genome projects in diverse species is growing, incomplete sequences of PL1O homologs still exist in the published genomes, especially for the challenges in sequencing and assembly of the $Y$ $\mathrm{Chr}$ due to its highly repetitive nature. Further understanding of the evolution of sex Chr linked genes largely relies on the clarification of the diverse genomes in species other than primates.

\section{Conclusion}

Our analyses revealed that several conserved putative PL10 homologous regions, in addition to the functional PL10/DDX $3 X / D D X 3 Y$, are present on the autosome and mammalian X Chr. These homologs share high similarity (> 70\%) and coverage (> 50\%) with mouse Pl10 but contain premature stop codons or indels, resulting in shorter putative peptides and/or frameshifts, suggesting their pseudogenization during the course of evolution $[27,48]$. The eutherian $D D X 3 X / D D X 3 Y$ located in XAR/YAR were derived from the translocation of the orthologs on the ancestral autosome [15]. The identified putative autosomal homologs in mammals in the present study were retroposed from the $D D X 3 X$ while the additional $\mathrm{X}$ homologs in primates were transposed from the $D D X 3 Y$. These translocation events are lineage-specific. Like the bovine PL10, these homologs may still be active transcriptionally. Positive selection appears to operate on the PL10 homologs during the evolution. In addition to the highly conserved domain regions, several sites in the non-domain regions of functional PL10 homologs may play roles in enhancing the gene function in a lineagespecific manner.

The results reported in this study not only increase our knowledge regarding the molecular evolution of PL1O homologs, which will facilitate the future functional characterization of PL10 homologs, but also provide a valuable model to investigate the origin and evolution of the mammalian sex Chrs and the mechanisms behind lineage-specific gene duplication and functionality.

\section{Methods}

\section{Sequence retrieval}

By blating [49] the mouse Pl10 mRNA sequence against the genomes in UCSC genome database [17], we detected several putative homologous regions in mammals, birds and amphibians. We retrieved the homologous sequences from UCSC and conducted the pairwise alignment by the Bl2seq (NCBI Blast package) program using the mouse Pl10 mRNA sequence as the subject to filter out the sequences which cover $<50 \%$ of the mouse Pl10. We excluded the homologous sequences for the species without clear chromosomal annotations from our study. In addition, we downloaded the PL1O homologous sequences for other species from the NCBI nucleotide database based on the literature and database mining [2]. Afterwards, we used blastx [50] to confirm the identity of each sequence and retrieved the corresponding entries from the NCBI when the query sequences matched these entries perfectly. Alternatively, for sequences without a perfect hit in the database, we used the collected genomic sequences for gene prediction using the GENSCAN [18]. The predicted peptides were used for blastp [50] analysis to clarify their identities. Whenever the predicted proteins can match to the entries in the database, we used the deposited sequences in our analyses. The results were summarized in Tables 1 and 2. Several purpose-designed scripts were coded in $\mathrm{C}++$ to facilitate the analysis.

\section{Phylogenetic analysis}

We performed multiple sequence alignments to investigate the conservation of the domain regions by ClustalW [51]. In order to ensure alignment quality, we first prealigned the annotated homologous sequences using their translated amino acid sequences in the coding regions. The parameters of the ClustalW for multiple alignment stage were modified to 3.0 for the gap opening penalty and 1.8 for the gap extension penalty to improve the alignment. Following that, we aligned the non-annotated homologous sequences based on the nucleotide sequences, which was further refined by manual adjustment. The positions of DEAD and the helicase domain were defined based on the annotation of Pfam [52] for the human $D D X 3 X$. The alignment was visualized through 
Jalview [53]. The degree of conservation was calculated by the empirical Bayesian method implemented in ConSurf [21] to investigate the highly conserved sites in the published human $D D X 3 X$ structures (PDB: 2I4I) covering the important interacting domains involved in the RNA metabolism of the PL1O related homologs [35]. The conserved degree was represented through different colors using Jmol [54] as shown in Fig. 4A. In addition, we used the alignment to establish the Neighbor-Joining (NJ) phylogenic tree to study the relationship between PL10, $D D X 3 X$, and $D D X 3 Y$ by MEGA4 [55] with the Maximum Composition Likelihood approach and 1000 bootstrap replicates (Fig. 1). The rate variation among sites was modeled with a gamma distribution (shape parameter = 0.91 , estimated by the Model Selection of TOPALi (version 2.5 [56]). In Fig. 1, the branches corresponding to partitions reproduced in less than $65 \%$ bootstrap replicates are collapsed. The reason for using the $\mathrm{NJ}$ method is that the average pairwise Jukes-Cantor (JC) distance of the dataset is 0.362 smaller than 1.0, which is suitable for making the NJ trees [57]. We applied the pairwise deletion to remove gaps as our sequence lengths are varied, and a complete removal of the gaps is not a good choice as it eliminates a large portion of phylogenetically meaningful sites from consideration. Further, we used the Maximum Composite Likelihood model, recommended by the author of MEGA4 as a better evolutionary model. We also used the PHYML [58] and MrBayes [59] implemented in TOPALi to generate the phylogenetic trees [Additional File 4 and File 5]. The models used in PHYML and MrBayes are general-time-reversible (GTR+Gamma) selected via the TOPALi.

Potential pseudogenes were excluded from the positive selection test since the selective constraint may not act on them anymore. We prepared an amino acid alignment and the corresponding cDNA alignment for the complete set of 25 sequences for the lineage test and a small dataset (14) containing only the functional mammalian PL10 homologs for the site-specific test. The Codeml [20] in PAML4 was applied for the following analyses. The sites and lineages subject to positive selection were detected based on the maximum likelihood approach [60]. We compared the log likelihood values $(l)$ derived from pairs of models to testify if there was a significant difference between model pairs by LRT. Each pair of the models contains a simple model, where the $\mathrm{Ka} / \mathrm{Ks}$ ratios of the sites are limited, and a complex model, where the $\mathrm{Ka} / \mathrm{Ks}$ ratios can be varied. We can infer the occurrence of lineage-specific and site-specific positive selection when the estimated $\mathrm{Ka} / \mathrm{Ks}$ ratio in the complex model is larger than 1.0 and the calculated test statistic $(2 \Delta l)$ is significantly larger than the critical values of the $X^{2}$ distribution at the corresponding degrees of freedom. We used M0 (oneratio) and M1 (free-ratio) to test whether the lineage-specific positive selection occurred. The pairs of models used for the site-specific test were M0 (One Ratio)/M3 (Discrete), M1a (Nearly Neutral)/M2a (Positive Selection), M7 (beta)/M8 (beta \& w) [20]. Each test was repeated to ensure the reproducible statistical results. The detailed assumptions and descriptions of each model were illustrated by Yang et al. [61,20].

\section{Additional material}

\begin{abstract}
Additional file 1 Selection test result for functional mammalian PL10
homologs. Log-likelihood and parametric estimates of the site-specific positive selection for functional mammalian PL10 homologs.

Additional file $\mathbf{2}$ Test statistics of site-specific positive selection test Likelihood ratio statistics $(2 \Delta l)$ of the site-specific positive selection test.

Additional file $\mathbf{3}$ The multiple alignment and conserved regions of the PL10 related sequences. Only the domain regions are shown in this figure. The newly identified homologs are shaded in grey while the DEADC and Helicase C-terminal conserved domain are boxed in red. The blue color residues indicate the sites with conserved identity over $85 \%$. The DDX3X/Y and PL10 specific insertion and the extended DDX3 unique positive residues are highlighted in green
\end{abstract}

Additional file 4 The Maximum-likelihood tree built for the PL10 related homologous sequences. The evolutionary tree was built based on the Maximum-likelihood method implemented in TOPALi [56]. The bootstrap values (1000 replicates) are shown next to the branches. The evolutionary model used was GTR+G. The tree has a similar topology to Fig. 1 Compared to Fig. 1, a swap occurred between the branches leading to the hydra, clamworm and flatworm homologs, and another swap observed between the branches leading to the putative rat Chr19 homologous region and other rodent homologous regions. The branches leading to the non-annotated autosomal homologous clusters of $P L 10$ in primate are highlighted in blue; the branches leading to the rodent $P / 10$ are highlighted in green; the branches leading to the non-annotated X-homologs are highlighted in red. The PL 10/DDX3X cluster and the DDX3Y cluster are marked by vertical lines on the right.

Additional file $\mathbf{5}$ The Bayesian inference tree built for the $P L 10$ related homologous sequences. The evolutionary tree was built based on the Bayesian inference method implemented in TOPALi [56]. The bootstrap values (1000 replicates) are shown next to the branches. The evolutionary model used was GTR+G. This tree is very much similar to the Supplementary Fig. 2.

Additional file 6 The multiple sequence alignment of the putative PL10 homologs. A total of 37 sequences from 19 species were included in the alignment. The alignment length is $3944 \mathrm{bp}$. The number of phylogenetic informative sites is 2954 (79.4\%).

\section{Authors' contributions}

TCC assisted in designing the study, collected the data, carried out the analyses and drafted the manuscript. WSL conceived of the study, participated in the experimental design and edited the manuscript. All authors read and approved the final manuscript.

\section{Acknowledgements}

We are grateful to Dr. Daniel Hagen, Department of Dairy and Animal Science at the Pennsylvania State University, for his valuable comments. This work was supported by grants from USDA-CSREES (No. 2005-35205-18653 and No. 201065205-20362) and start-up funds from the Pennsylvania State University to Liu, W-S.

\section{Author Details}

1Department of Dairy and Animal Science, The Center for Reproductive Biology and Health (CRBH), College of Agricultural Sciences, The Pennsylvania State University, University Park, PA, 16802, USA and ${ }^{2}$ The Integrative

Biosciences Program, Bioinformatics and Genomics Option, The Huck Institute of Life Sciences, The Pennsylvania State University, University Park, PA, 16802,

USA

Received: 12 October 2009 Accepted: 3 May 2010

Published: 3 May 2010 


\section{References}

1. Leroy P, Seboun E, Mattei MG, Fellous M, Bishop CE: Testis-specific transcripts detected by a human Y-DNA-derived probe. Development 1987, 101(Suppl):177-183.

2. Rosner A, Rinkevich B: The DDX3 subfamily of the DEAD box helicases: divergent roles as unveiled by studying different organisms and in vitro assays. Curr Med Chem 2007, 14:2517-25

3. Vong QP, Li Y, Lau YC, et al:: Structural characterization and expression studies of Dby and its homologs in the mouse. J Androl 2006, 27:653-61.

4. UniProt [http://www.uniprot.org/]

5. Linder P: Dead-box proteins: a family affair--active and passive players in RNP-remodeling. Nucleic Acids Res 2006, 34:4168-80,

6. Soulat D, Bürckstümmer T, Westermayer S, et al:: The DEAD-box helicase DDX3X is a critical component of the TANK-binding kinase 1dependent innate immune response. EMBO J 2008, 27:2135-2146.

7. Botlagunta M, Vesuna F, Mironchik Y, et al:: Oncogenic role of DDX3 in breast cancer biogenesis. Oncogene 2008, 27:3912-3922.

8. Rosner A, Paz G, Rinkevich B: Divergent roles of the DEAD-box protein BS-PL10, the urochordate homologue of human DDX3 and DDX3Y proteins, in colony astogeny and ontogeny. Dev Dyn 2006, 235:1508-1521.

9. Kwong $A D$, Rao $B G$, Jeang K: Viral and cellular RNA helicases as antivira targets. Nat Rev Drug Discov 2005, 4:845-853.

10. Kuo $P$, Lin $Y$, Teng $Y$, et al.: Transcriptional levels of four $Y$ chromosomelinked AZF genes in azoospermic men and their association with successful sperm retrieval. Urology 2004, 63:131-6. discussion 136.

11. Foresta $C$, Moro E, Ferlin A: Prognostic value of $Y$ deletion analysis. The role of current methods. Hum Reprod 2001, 16:1543-7.

12. Lardone MC, Parodi DA, Valdevenito R, et al.: Quantification of DDX3Y, RBMY1, DAZ and TSPY mRNAs in testes of patients with severe impairment of spermatogenesis. Mol Hum Reprod 2007, 13:705-712.

13. Mazeyrat S, Saut N, Sargent CA, et al:: The mouse Y chromosome interval necessary for spermatogonial proliferation is gene dense with syntenic homology to the human AZFa region. Hum Mol Genet 1998, 7:1713-24.

14. Liu W, Wang A, Yang Y, et al:: Molecular characterization of the DDX3Y gene and its homologs in cattle. Cytogenet and Genome Res 2009.

15. Wilson MA, Makova KD: Evolution and survival on eutherian sex chromosomes. PLoS Genet 2009, 5:e1000568.

16. NCBI [http://www.ncbi.nlm.nih.gov/]

17. UCSC Genome Browser Home [http://genome.ucsc.edu/

18. Burge $C$, Karlin S: Prediction of complete gene structures in human genomic DNA. J Mol Biol 1997, 268:78-94.

19. Ensembl Genome Browser [http://www.ensembl.org/index.html]

20. Yang Z: PAML 4: phylogenetic analysis by maximum likelihood. Mol Biol Evol 2007, 24:1586-1591.

21. Landau M, Mayrose I, Rosenberg Y, et al:: ConSurf 2005: the projection of evolutionary conservation scores of residues on protein structures. Nucleic Acids Res 2005, 33:W299-302.

22. RCSB Protein Data Bank [http://www.rcsb.org/pdb/home/home.do]

23. Bachtrog D: A dynamic view of sex chromosome evolution. Curr Opin Genet Dev 2006, 16:578-85.

24. Graves JAM: Sex chromosome specialization and degeneration in mammals. Cell 2006, 124:901-14.

25. Wallis MC, Waters PD, Graves JAM: Sex determination in mammals-before and after the evolution of SRY. Cell Mol Life Sci 2008, 65:3182-3195.

26. Marshall Graves JA: Human $Y$ chromosome, sex determination, and spermatogenesis- a feminist view. Biol Reprod 2000, 63:667-676.

27. Emerson JJ, Kaessmann $H$, Betrán E, Long M: Extensive gene traffic on the mammalian X chromosome. Science 2004, 303:537-540.

28. Betrán E, Thornton K, Long M: Retroposed New Genes Out of the $\mathrm{X}$ in Drosophila. Genome Research 2002, 12:1854-1859.

29. Betran E, Wang W, Jin L, Long M: Evolution of the Phosphoglycerate mutase Processed Gene in Human and Chimpanzee Revealing the Origin of a New Primate Gene. Mol Biol Evol 2002, 19:654-663.

30. Brosius J: RNAs from all categories generate retrosequences that may be exapted as novel genes or regulatory elements. Gene 1999, 238:115-134.

31. Hedges SB, Dudley J, Kumar S: TimeTree: a public knowledge-base of divergence times among organisms. Bioinformatics 2006, 22:2971-2972.
32. Korneev SA, Park JH, O'Shea M: Neuronal expression of neural nitric oxide synthase (nNOS) protein is suppressed by an antisense RNA transcribed from an NOS pseudogene. J Neurosci 1999, 19:7711-7720.

33. Balakirev ES, Ayala FJ: Pseudogenes: are they "junk" or functional DNA? Annu Rev Genet 2003, 37:123-51.

34. Gerrard DT, Filatov DA: Positive and negative selection on mammalian $Y$ chromosomes. Mol Biol Evol 2005, 22:1423-1432.

35. Högbom M, Collins R, Berg S van den, et al.: Crystal structure of conserved domains 1 and 2 of the human DEAD-box helicase DDX3X in complex with the mononucleotide AMP. J Mol Biol 2007, 372:150-159.

36. Shi H, Cordin O, Minder CM, Linder P, Xu R: Crystal structure of the human ATP-dependent splicing and export factor UAP56. Proc Natl Acad Sci USA 2004, 101:17628-17633.

37. Yan X, Mouillet J, Ou Q, Sadovsky Y: A novel domain within the DEADbox protein DP103 is essential for transcriptional repression and helicase activity. Mol Cell Biol 2003, 23:414-423.

38. Yagüe J, Alvarez I, Rognan D, et al:: An N-acetylated natural ligand of human histocompatibility leukocyte antigen (HLA)-B39. Classical major histocompatibility complex class I proteins bind peptides with a blocked NH(2) terminus in vivo. J Exp Med 2000, 191:2083-2092.

39. Rush J, Moritz A, Lee KA, et al.: Immunoaffinity profiling of tyrosine phosphorylation in cancer cells. Nat Biotechnol 2005, 23:94-101.

40. Tao WA, Wollscheid B, O'Brien R, et al:: Quantitative phosphoproteome analysis using a dendrimer conjugation chemistry and tandem mass spectrometry. Nat Methods 2005, 2:591-598.

41. Molina H, Horn DM, Tang N, Mathivanan S, Pandey A: Global proteomic profiling of phosphopeptides using electron transfer dissociation tandem mass spectrometry. Proc Natl Acad Sci USA 2007, 104:2199-2204.

42. Yu L, Zhu Z, Chan KC, et al:: Improved titanium dioxide enrichment of phosphopeptides from HeLa cells and high confident phosphopeptide identification by cross-validation of MS/MS and MS/MS/MS spectra. $J$ Proteome Res 2007, 6:4150-4162.

43. Dephoure N, Zhou C, Villén J, et al:: A quantitative atlas of mitotic phosphorylation. Proc Natl Acad Sci USA 2008, 105:10762-10767.

44. Brill LM, Salomon AR, Ficarro SB, et al:: Robust phosphoproteomic profiling of tyrosine phosphorylation sites from human T cells using immobilized metal affinity chromatography and tandem mass spectrometry. Anal Chem 2004, 76:2763-2772

45. Daub H, Olsen JV, Bairlein M, et al: Kinase-selective enrichment enables quantitative phosphoproteomics of the kinome across the cell cycle. Mol Cell 2008, 31:438-448.

46. Cao L, Yu K, Banh C, et al:: Quantitative time-resolved phosphoproteomic analysis of mast cell signaling. J Immunol 2007 179:5864-5876

47. Ballif BA, Carey GR, Sunyaev SR, Gygi SP: Large-scale identification and evolution indexing of tyrosine phosphorylation sites from murine brain. J Proteome Res 2008, 7:311-318.

48. Majumdar M, Bharadwaj A, Ghosh I, Ramachandran S, Datta K: Evidence for the Presence of HABP1 Pseudogene in Multiple Locations of Mammalian Genome. DNA Cell Bio 2002, 21:727-735.

49. Kent WJ: BLAT--the BLAST-like alignment tool. Genome Res 2002, 12:656-664

50. BLAST: Basic Local Alignment Search Tool http:// blast.ncbi.nlm.nih.gov/Blast.cgi]

51. Larkin MA, Blackshields G, Brown NP, et al:: Clustal W and Clustal X version 2.0. Bioinformatics 2007, 23:2947-2948.

52. Finn RD, Tate J, Mistry J, et al.: The Pfam protein families database. Nucleic Acids Res 2008, 36:D281-288.

53. Waterhouse AM, Procter JB, Martin DMA, Clamp M, Barton GJ: Jalview Version 2--a multiple sequence alignment editor and analysis workbench. Bioinformatics 2009, 25:1189-1191.

54. Jmol: an open-source Java viewer for chemical structures in 3D [http:/ /imol.sourceforge.net//

55. Tamura K, Dudley J, Nei M, Kumar S: MEGA4: Molecular Evolutionary Genetics Analysis (MEGA) software version 4.0. Mol Biol Evol 2007, 24:1596-1599.

56. Milne I, Lindner D, Bayer M, et al.: TOPALi v2: a rich graphical interface for evolutionary analyses of multiple alignments on HPC clusters and multi-core desktops. Bioinformatics 2009, 25:126-127. 
57. Nei M, Kumar S: Molecular Evolution and Phylogenetics 1st edition. Oxford University Press, USA; 2000.

58. Guindon S, Gascuel O: A simple, fast, and accurate algorithm to estimate large phylogenies by maximum likelihood. Syst Biol 2003, 52:696-704

59. Ronquist F, Huelsenbeck JP: MrBayes 3: Bayesian phylogenetic inference under mixed models. Bioinformatics 2003, 19:1572-1574.

60. Yang Bielawski: Statistical methods for detecting molecular adaptation. Trends Ecol Evol (Amst) 2000, 15:496-503.

61. Yang Z, Swanson WJ, Vacquier VD: Maximum-likelihood analysis of molecular adaptation in abalone sperm lysin reveals variable selective pressures among lineages and sites. Mol Biol Evol 2000, 17:1446-1455.

62. Felsenstein J: Confidence Limits on Phylogenies: An Approach Using the Bootstrap. Evolution 1985, 39:791-783.

63. Tamura K, Nei M, Kumar S: Prospects for inferring very large phylogenies by using the neighbor-joining method. Proc Natl Acad Sci USA 2004, 101:11030-11035.

64. Laskowski RA: PDBsum new things. Nucleic Acids Res 2009, 37:D355-359.

doi: $10.1186 / 1471-2148-10-127$

Cite this article as: Chang and Liu, The molecular evolution of PL10

homologs BMC Evolutionary Biology 2010, 10:127

Submit your next manuscript to BioMed Central and take full advantage of:

- Convenient online submission

- Thorough peer review

- No space constraints or color figure charges

- Immediate publication on acceptance

- Inclusion in PubMed, CAS, Scopus and Google Scholar

- Research which is freely available for redistribution

Submit your manuscript at www.biomedcentral.com/submit
C) Biomed Central 